Int. J. Electrochem. Sci., 12 (2017) $2184-2193$

\title{
Electrochemical Behaviors of Myoglobin on Ionic Liquid- Graphene-Cobalt Oxide Nanoflower Composite Modified Electrode and Its Electrocatalytic Activity
}

Sheng Kang, Wenshu Zhao, Xiaoyan Li, Zuorui Wen, Xueliang Niu, Bolin He, Linfang Li, Wei Sun*

Key Laboratory of Tropical Medicinal Plant Chemistry of Ministry of Education, College of Chemistry and Chemical Engineering, Hainan Normal University, Haikou 571158, P R China, *E-mail: swyy26@hotmail.com

doi: $10.20964 / 2017.03 .64$

Received: 18 December 2016 / Accepted: 29 January 2017 / Published: 12 February 2017

A biocomposite was prepared by mixing myoglobin (Mb), ionic liquid (IL) 1-butyl-3-methylimidazolium tetrafluoroborate, graphene (GR) and cobalt oxide $\left(\mathrm{Co}_{3} \mathrm{O}_{4}\right)$ nanoflower together. Then the mixture was applied on carbon ionic liquid electrode (CILE) to get a modified electrode with chitosan film. Ultraviolet-visible and FT-IR spectroscopic experiments showed that $\mathrm{Mb}$ in the composite remained its native structure. On cyclic voltammogram a pair of well-defined redox peaks was got in $0.1 \mathrm{~mol} \mathrm{~L}^{-1} \mathrm{pH} 5.0$ phosphate buffer solution, which was ascribed to the realization of direct electrochemistry of $\mathrm{Mb}$ with the underlying electrode. The synergistic effects of IL, GR and $\mathrm{Co}_{3} \mathrm{O}_{4}$ nanoflower provided a fast electron transfer path for the movement of electron from $\mathrm{Mb}$ active centers to the electrode. Electrochemical parameters of electrode reaction of $\mathrm{Mb}$ were calculated. The $\mathrm{Mb}$ modified electrode showed excellent electrocatalytic ability towards the reduction of trichloroacetic acid, which indicated that a new third-generation electrochemical biosensor was constructed successfully.

Keywords: Myoglobin; Ionic liquid; Graphene; Cobalt oxide nanoflower; Electrochemical biosensor

\section{$\underline{\text { FULL TEXT }}$}

(C) 2017 The Authors. Published by ESG (www.electrochemsci.org). This article is an open access article distributed under the terms and conditions of the Creative Commons Attribution license (http://creativecommons.org/licenses/by/4.0/). 